\title{
Study for s-process using neutron beam provided from ANNRI of J-PARC
}

\author{
Takehioto Hayakawa $^{12}$, Yosuke Toh ${ }^{1}$, Akira Kimura ${ }^{1}$, Shoji Nakamura ${ }^{1}$, Toshiyuki Shizuma ${ }^{1}$ and Hideo \\ Harada $^{1}$ \\ ${ }^{1}$ Japan Atomic Energy Agency, Tokai, Ibaraki, Japan \\ ${ }^{2}$ Natonal Astronomical Observatory of Japan, Mitaka, Tokyo, Japan
}

\begin{abstract}
Most isotopes heavier than iron are synthesized by the slow neutron capture reaction process (s-process) in stars. Isomers in stable isotopes have sometimes an important role as a branching point in nucleosynthesis flow in the s-process. An isomer with a half-life of $14.1 \mathrm{y}$ in ${ }^{113} \mathrm{Cd}$ is a branching point from which a nucleosynthesis flow reaches to a rare isotope ${ }^{115} \mathrm{Sn}$. The astrophysical origin of ${ }^{115} \mathrm{Sn}$ has remained still an open question. The s-process abundance of ${ }^{115} \mathrm{Sn}$ depends on the ratio of the ${ }^{112} \mathrm{Cd}(\mathrm{n}, \gamma){ }^{113} \mathrm{Cd}^{\mathrm{m}}$ reaction cross section to the ${ }^{112} \mathrm{Cd}(\mathrm{n}$, $\gamma)^{113} \mathrm{Cd}^{\mathrm{gs}}$ reaction cross section. However, the isomer production ratio following the neutron capture reaction has not been measured in the energy region higher than the thermal energy. An intense neutron beam experimental system, ANNRI, in J-PARC has a high purity germanium (HPGe) detector system consisting of two cluster detectors. We have measured $\gamma$-rays decaying to the ground state and the isomer using the HPGe detectors in conjunction with a time-offlight method at ANNRI.
\end{abstract}

\section{Introduction}

Nuclides heavier than iron were predominantly synthesized by two stellar neutron induced reactions of slow neutron capture reactions (s-process) and rapid neutron capture reaction process (r-process). The astrophysical origin of rare isotopes in Cd-In-Sn region, ${ }^{113} \mathrm{In},{ }^{114} \mathrm{Sn}$ and ${ }^{115} \mathrm{Sn}$, have been a longstanding problem [1-3]. These isotopes are classified to "p-nuclei" which have following features. Their isotopic fractions are typically lower than $1 \%$. They are neutron-deficient isotopes. They are located on the outside of the main path of the s-process and shielded against the $\beta$-decay after the freezeout of the r-process by stable isobars. Hayakawa et al. [4] found an empirical relationship between a p-nucleus and an s-nucleus with the same atomic number in the solar abundances. This relationship is evidence that the most probable origin of $27 \mathrm{p}$-nuclei is photodisintegration reactions in supernova explosions ( $\gamma$-process). However, the origin of odd-N isotope, ${ }^{115} \mathrm{Sn}$, has remained still an open question, since the relationship cover only the even-N nuclei.

Isomers in stable isotopes have sometimes an important role as a branching point in nucleosynthesis flow in the s-process (for examples, see refs. [3,5]). As shown in Fig. 1, $\beta$-unstable isomers in stable isotopes have important role to synthesis these three p-nuclei. The p-nuclei, ${ }^{113} \mathrm{In}$ and ${ }^{115} \mathrm{Sn}$, may be produced through isomers in ${ }^{113} \mathrm{Cd}$ and ${ }^{115} \mathrm{In}$, respectively, by the $\beta$-decay after the $\mathrm{r}$ process and/or weak branches of the s-process. The nucleus ${ }^{115} \mathrm{Sn}$ may be produced by a weak branch of the s-process (see the dotted arrows in Fig. 1). The neutron capture reaction cross-sections were 
systematically measured and the resonance parameters were also evaluated [6]. However, the partial neutron capture cross section to ${ }^{113} \mathrm{Cd}^{\mathrm{m}}$ has not been measured except thermal energy. The abundance of ${ }^{115} \mathrm{Sn}$ depends on the population ratio of ${ }^{113} \mathrm{Cd}^{\mathrm{m}}$ to ${ }^{113} \mathrm{Cd}^{\mathrm{gs}}$ following neutron capture reactions on ${ }^{112} \mathrm{Cd}$. It was suggested that the population of the isomer in ${ }^{113} \mathrm{Cd}$ should follow by thermalequilibrium conditions in s-process environments of $k T=8-30 \mathrm{keV}$. In such cases, the population ratio, $\mathrm{m} /(\mathrm{m}+\mathrm{gs})$, depend only on the temperature of the environment where the s-process occurs.

Hayakawa et al. [3] measured the neutron capture cross-section to ${ }^{113} \mathrm{Cd}^{\mathrm{m}}$ using thermal neutrons provided by a nuclear research reactor JRR-3. To estimate the ratio of ${ }^{112} \mathrm{Cd}(\mathrm{n}, \gamma){ }^{112} \mathrm{Cd}^{\mathrm{m}}$ to ${ }^{112} \mathrm{Cd}(\mathrm{n}$, $\gamma)^{112} \mathrm{Cd}^{\mathrm{m}}+{ }^{112} \mathrm{Cd}(\mathrm{n}, \gamma){ }^{112} \mathrm{Cd}^{\mathrm{gs}}$ at the s-process energies of $5-50 \mathrm{keV}$ the ratio as a function of the neutron incident energy was calculated by using a statistical model. It was found that the $\mathrm{m} /(\mathrm{m}+\mathrm{gs})$ ratio depends strongly on branching ratios of $\gamma$-decay of excited states. The calculated ratios are almost constant over a wide range of $\mathrm{E}<100 \mathrm{keV}$. The s-process contribution to ${ }^{115} \mathrm{Sn}$ using a classical "steady flow" model with the evaluated ratio at stellar temperatures was evaluated. It was obtained that $3-4 \%$ of the ${ }^{115} \mathrm{Sn}$ solar abundance may originate from the s-process. However, the $\mathrm{m} /(\mathrm{m}+\mathrm{gs})$ ratios depend on the detailed nuclear structure and thus $\mathrm{m} /(\mathrm{m}+\mathrm{gs})$ ratios in an energy region of 5-50 keV should be experimentally measured. Thus, we have measured $\gamma$-rays using the HPGe detectors in conjunction with a time-of-flight method at an intense neutron beam experimental system, ANNRI, in J-PARC.

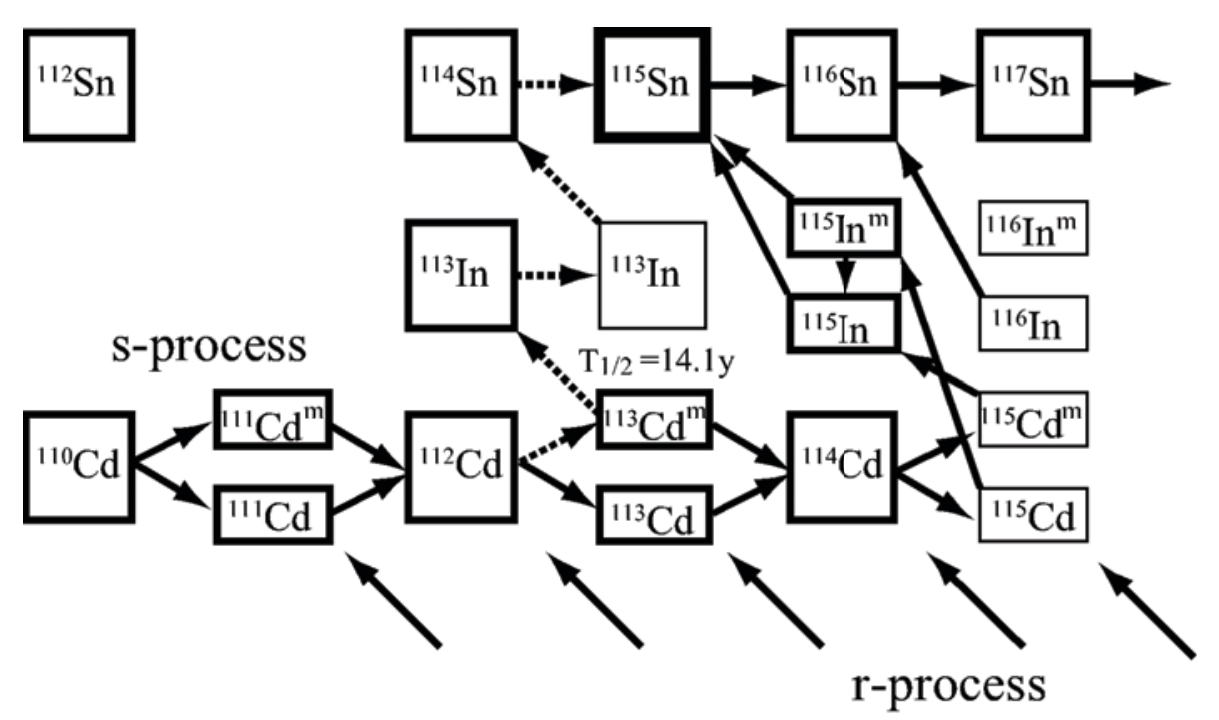

Figure 1. Partial nuclear chart around ${ }^{115} \mathrm{Sn}$ and nucleosynthesis flows.

\section{Experimental producers}

The experiment was performed using Accurate Neutron-Nucleus Reaction measurement Instrument (ANNRI), installed at a neutron beam line, BL04, in the Materials and Life Science Experimental Facility (MLF) in the Japan Proton Accelerator Research Complex (J-PARC) [7]. A pulsed proton beam with an energy of $3 \mathrm{GeV}$ was injected into a mercury target of the spallation source in MLF. The proton beam was accelerated with a beam power of $200 \mathrm{~kW}$ and at a repetition rate of $25 \mathrm{~Hz}$. Neutrons were emitted from the mercury target by spallation reactions. The proton beam was operated with the double bunch mode; A single bunch includes two proton pulses with pulse widths of $100 \mathrm{~ns}$ and with an time interval between the two pulses of $600 \mathrm{~ns}$. Thus, the time resolution was determined by the double pulse mode. The neutron beam collimator with a diameter of $7 \mathrm{~mm}$ was used, which was located upstream from the target position. The diameter of the neutron beam on the target is $7 \mathrm{~mm}$. 
A lead absorber with a thickness of $37.5 \mathrm{~mm}$ was located downstream from the neutron source to absorb prompt $\gamma$-rays generated by the spallation reaction.

The ANNRI has a large highly pure germanium (HPGe) detector array, $4 \pi$ Ge spectrometer, which consists of two cluster HPGe detectors, eight coaxial HPGe detectors, and BGO Compton-suppression detectors. In the present experiment, the two cluster HPGe detectors were used. This detector system was located $21.5 \mathrm{~m}$ downstream from the neutron source in the MLF. The $\gamma$-rays were recorded when at least two HPGe crystals detected photons. The energy of a neutron was measured using a Time-ofFlight (TOF) method by recording the time when a detector is fired. The energies of the $\gamma$-rays and a neutron were recorded in event by event as the list mode. $\mathrm{A}{ }^{112} \mathrm{Cd}$ foil enriched to $98.27 \%$ was used as the target.

The energy difference between the ground state and the isomer is only $264 \mathrm{keV}$ and thus it is difficult to distinguish two decays to the ground state or the isomer following neutron capture reactions using standard large volume scintillation detectors such as $\mathrm{NaI}(\mathrm{Tl})$. Thus, in the present experiment, we measured relative ratios of $\gamma$-rays decaying to the ground state to that to the isomer using the HPGe detectors.

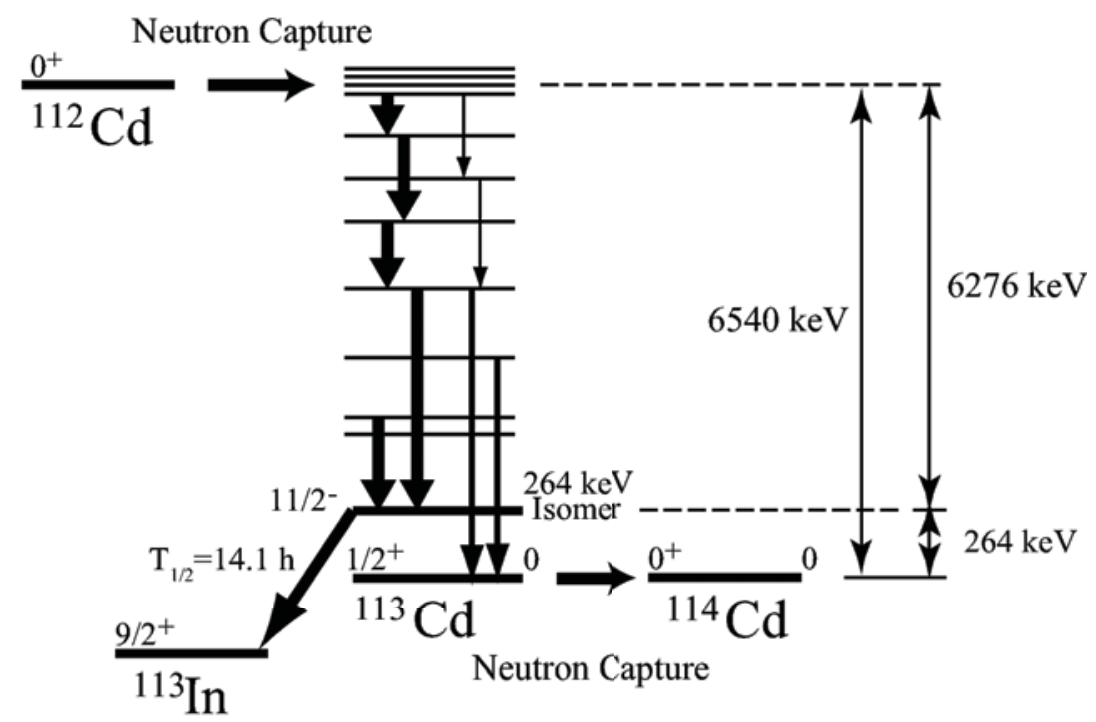

Figure 2. Partial level scheme of ${ }^{115} \mathrm{Sn}$.

\section{Results and Discussion}

Figure 3 shows a TOF spectrum higher than the thermal energy. In the thermal energy region, the large peak originated from ${ }^{113} \mathrm{Cd}$ is observed. This ${ }^{113} \mathrm{Cd}$ is inferred to originate from the contamination to the ${ }^{112} \mathrm{Cd}$ enriched target. The fraction of $113 \mathrm{Cd}$ is only $0.6 \%$. The thermal neutron capture cross-section of ${ }^{113} \mathrm{Cd}$ is 20650 barn, whereas that of ${ }^{112} \mathrm{Cd}$ is considered to be lower than 2 barn. As discussed in the previous paper [3], the thermal neutron capture cross section of ${ }^{112} \mathrm{Cd}$ has not been measured yet. As shown in Fig. 3, the relatively strong resonances like $66.8 \mathrm{eV}, 227 \mathrm{eV}$, and 440 $\mathrm{eV}$, are observed. This suggests that $\gamma$-rays of ${ }^{112} \mathrm{Cd}$ is dominant in the energy region higher than $\mathrm{eV}$.

Figure 4 shows a $\gamma$-ray energy spectrum gated by the 66.8 -eV neutron energy in TOF. The $\gamma$-rays decaying to the ground state with energies of 299 and $316 \mathrm{keV}$ are clearly observed. A $\gamma$-ray of 259 $\mathrm{keV}$ is also observed, which decays to the isomer on ${ }^{113} \mathrm{Cd}$. In this way, the present experiment 
suggests that it is possible to evaluate the isomer production ratio to the ground state as a function of neutron energy with high statics.

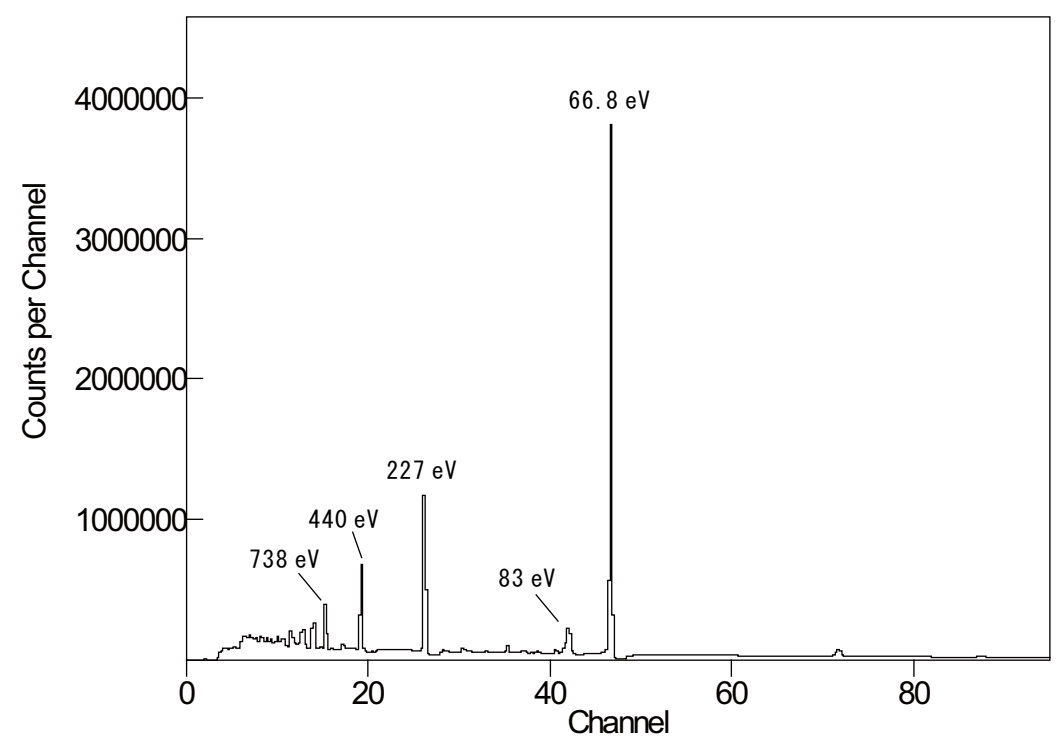

Figure 3. TOF spectrum on the ${ }^{112} \mathrm{Cd}$ enriched target.

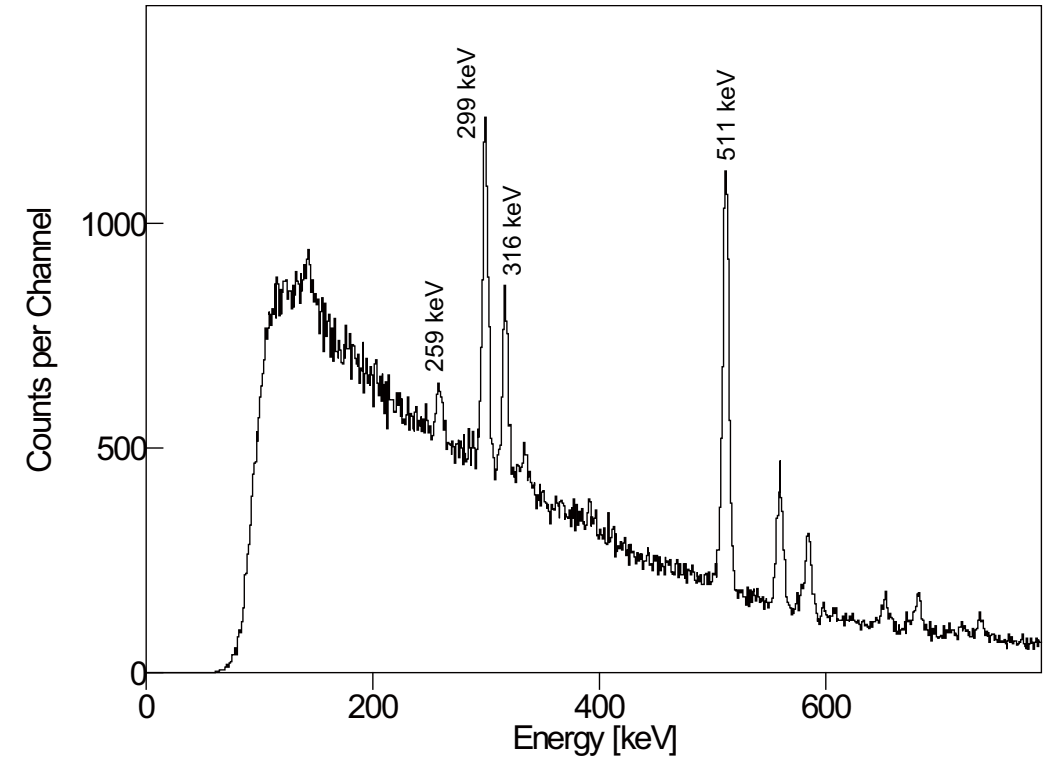

Figure 4. Partial nuclear chart around ${ }^{115} \mathrm{Sn}$ and nucleosynthesis flows.

\section{Summary}

The astrophysical origin of ${ }^{115} \mathrm{Sn}$ has remained still an open question. An isomer with a half-life of $14.1 \mathrm{y}$ in ${ }^{113} \mathrm{Cd}$ is a branching point from which a nucleosynthesis flow reaches to a rare isotope ${ }^{115} \mathrm{Sn}$. The s-process abundance of ${ }^{115} \mathrm{Sn}$ depends on the ratio of the ${ }^{112} \mathrm{Cd}(\mathrm{n}, \gamma){ }^{113} \mathrm{Cd}^{\mathrm{m}}$ reaction cross section to the ${ }^{112} \mathrm{Cd}(\mathrm{n}, \gamma){ }^{113} \mathrm{Cd}^{\mathrm{gs}}$ reaction cross section. However, the isomer production ratio following the neutron capture reaction has not been measured in the energy region higher than the thermal energy. 
We have measured $\gamma$-rays following neutron capture reactions on ${ }^{112} \mathrm{Cd}$ using the HPGe detectors in conjunction with a time-of-flight method at ANNRI. In the neutron energy region higher than $\mathrm{eV}$, we observed decay $\gamma$-rays to the ground state and the isomer. The present result suggests that it is possible to evaluate the isomer production ratio to the ground state as a function of neutron energy higher than $\mathrm{eV}$ with high statics.

\section{References}

1. R. A. Ward and H. Beer, Astrophy. Astron. 103, 189A (1981).

2. Ch. Theis, et al., Astrophy. J. 500, 1039M (1998).

3. T. Hayakawa et al., Astrophy. J. 707, 859 (2009).

4. T. Hayakawa et al., Phys. Rev. Lett. 93, 161102 (2004).

5. T. Hayakawa et al., Astrophy. J. 628, 533 (2005).

6. K. Volev, et al., Nucl. Instr. Method Phys. Res. B 300, 11 (2013).

7. K. Kino, et al., Nucl. Instr. Method Phys. Res. A 626, 58 (2011). 